\title{
SUCCESS RATE OF STRABISMUS SURGERY IN ADULT PATIENTS
}

\author{
Jasmina Alajbegović- Halimić ${ }^{1}$, Nina Jovanović², Tarik Halimić ${ }^{3}$
}

(C) 2019 by Acta Medica Saliniana

ISSN 0350-364X

DOI: $10.5457 / 505$

Jasmina Alajbegović- Halimić ${ }^{1}$, Nina Jovanović ${ }^{2}$ Tarik Halimić ${ }^{3}$

\section{ABSTRACT}

Background Purpose. Adults as well as children may have problems with strabismus, which can significantly affects on quality of social and professional life. Strabismus surgery in adults are not conditioned for years of a patient, and the main goal of this kind of surgery is to improve motoric-senzorial relationship of eyes, and to improve esthetic moment of a patient.

Patients\&Methods. A surgical review was performed on 35 adult patients with strabismus in period between January 2016 - May 2018 years. The type of strabismus, preoperative measurements, and indications for surgery were obtained from the patient notes, as were all final outcomes. Final measurements, on average, were 3 to 6 weeks postoperatively, and patients were asked to comment subjectively on their outcome.

Results. In total number 35 of adult strabismic patients, were $12(34,3 \%)$ male and $23(65,7 \%)$ female, mean age $34,15 \pm 13$ ( 20 to 67 years), 17 patiens $(48,6 \%$ ) were exotropic, esotropic $11(31,4 \%)$ patiens, and hypertropic $7(20 \%)$ patients. Indication for surgery in the most cases were aesthetic 28 (80\%). The success rate is measured by number of orthophoric patients with less than 10 prism diopters $31(88,6 \%)$, stereoacuity $22(62,9 \%)$ and patient satisfaction $30(85,7 \%)$.

Conclusion. Adult strabismus surgery is very successful and provides patients with a good level of satisfaction. Many patients seek surgical correction for cosmetic and psychosocial benefits.

Keywords: adult, strabismus, success, surgery

\section{INTRODUCTION}

Strabismus or eye misalignment is both a medical as well as psychosocial problem.

Medical(functional)problems involve: asthenopia, diplopia, suppression, limitation or lack of fusion and stereopsis, torticolis and possible monocular and binocular changes of vision.

Psychological and social problems are sometimes even greater than medical problems because patients with strabismus may experience a handicap in everyday life and in communication and sometimes limited capability for work, driving or sports. It is a common misconception that strabismus is a exclusively a childhood disease, but it is a common problem in adult population, approximately $4 \%$ of patients older than 15 years have strabismus $(1,2,3,4)$. Adult patients hardly make a decision for operation because they think they are either too old or they think that operation will make their condition more difficult, especially if patients have strabismus since childhood $(1,2,3)$.

There are two forms of strabismus in adult patients. The first form is strabismus since childhood which has not been treated or the treatment was unsuccessful. Certain forms of uncompensated phorias fall into this group. Some patients have good visual function, but they also have problems with binocular vision and some patients have amblyopia since childhood, in these cases it is important to emphasize that operation will not improve vision, but 
will only correct position of eyes. The second form is seen in adult age with multiple reasons such as trauma, stroke, thyroid orbitopathy, cranial nerve palsy, orbit and eye surgery. In these cases it is important to cure the primary disease $(1,2,3,4,5,6)$.

Strabismus treatment can be conservative and surgical. Conservative treatment includes prisms and orthoptic exercises and most of adult patients decide for this kind of treatment and botulinum toxin as well. Surgical treatment is indicated because of cosmetic and function: better appearance, fusion and stereopsis reparation, asthenopic problems, convergence improvement, elimination of diplopia or correction of ocular torticolis. Strabismus surgery is easier when patients are than children for the surgeon, because it does not have to be performed in general anaesthesia, it can be done in local anaesthesia, there are no parents demands and finally, the result is evident right after the surgery and the physician does not have to wait for patient to grow up and operator has more freedom for recession and resection of the muscles $(2,3,4)$.

The postoperative recovery is relatively short, only a few days and after that patients can return to their everyday activities. There is always a chance for a repeated procedure in case the patient is not satisfied. Patient must always get an explanation of benefits and risks of proceudure. Adult patients with chronic strabismus have been thought to have the potential to achieve only a cosmetic result from surgery. On the contrary, several studies have shown that unexpected sensory fusion is possible after excellent postoperative motor alignment in these patients $(5,6,7,8,9)$. Other functional benefits of strabismus surgery in the adult patient include the expansion of binocular visual fields in patients with esotropia and the psychosocial and economic benefits from restoration of ocular alignment (2). Potential risks are postoperative acquired diplopia, loss of fusion and repeated surgeries $(1,2,3)$.

\section{PATIENTS AND METHODS}

A retrospective chart review of all adult patients who had undergone strabismus surgery between January 2016 and May 2018 was performed. Patient selection criteria were adult patients with untreated or uncompleted treatment strabismus in childhood, other forms of strabismus we excluded. Final measurements were, on average, taken at 3 to 6 weeks postoperatively. These patients were consented to be included in the study, in accordance to the Helsinki Convention. Patients were asked to comment subjectively on their surgical outcome as very satisfied, satisfied, neutral, unsatisfied, or very dissatisfied. All of patients preoperative were evaluated with cover test, motility, convergence, preoperative and postoperative angle deviation with prisms dioptres (PD), fusion, stereopsis preoperative and postoperative with Titmus stereo testing, Prism adaptation testing and diplopia testing by Hess-Lancaster test. Statistic analysis is demonstrated with tables and charts, a descriptive statistical method including absolute and relative number of cases, mean value with standard deviation, median and interquartile range. Analysis was done using the statistical program for biomedical research MedCalc v 12.7 (Antwerp, Belgium).

\section{RESULTS}

In total $35(\mathrm{~N}=35)$ patients were included, $12(34,3 \%)$ patients were male and $23(65,7 \%)$ were female patients. Mean age was $34,5 \pm 13$ years (20-67 years). Central tendency analysis shows that most patients were 23 to 42 years old $(50 \%)$ with median age of 32 years.

Exotropia $17(48,6 \%)$ was more frequent than esotropia 11 ( 31,4\%), and hypertropia 7 (20\%.) cases. Amblyopia was present in 16 (45,7\%) cases, amblyopia was also more frequent on the left eye in 21 cases $(60 \%$ ).Table 1.

Table 1. Type of strabismus and amblyopia present in adult patients

Type of strabismus \& Amblyopia present Eye affected by amblyopia

$\begin{array}{lcccccc} & \mathrm{N} & (\%) & \mathrm{N} & (\%) & \text { Eye } & \mathrm{N}(\%) \\ \text { Esotropia } & 11 & 31,4 & \text { No } 19 & 54,3 & \text { Left eye } & \mathbf{2 1}(60 \%) \\ \text { Exotropia } & 17 & 48,6 & \text { Yes } 16 & 45,7 & \text { Right eye } & \mathbf{1 4}(40 \%) \\ \text { Hypertropia } & 7 & \mathbf{2 0 , 0} & & & & \\ \text { Total } & 35 & \mathbf{1 0 0 , 0} & \text { Total } & 35 & \mathbf{1 0 0 , 0} \text { Total } & 35(100 \%)\end{array}$

Most common indication for operative procedure was aesthetics, total of 28 cases (8o,o\%). Table 2. 
Table 2. Indication for adult strabismus surgery

\section{Indication for surgery}

\begin{tabular}{|lcc|}
\hline Asthenopia & N & $\%$ \\
\hline Torticolis & 1 & 2,86 \\
\hline Diplopia & 4 & 11,43 \\
\hline Aesthetic/psychological & 2 & 5,71 \\
Total & 28 & 80,0 \\
\hline
\end{tabular}

Success rate was defined as an alignment that was within 10 prism diopters of straight with no subjective symptoms of diplopia or visual confusion after one strabismus surgical procedure, good steropsis ( 40 to 60 seconds of arc) and patient satisfaction.

Preoperative angle mean value was $20,5^{\circ} \pm 11,9^{\circ}\left(5^{\circ}\right.$ $50^{\circ}$ ), postoperative angle mean value was $2,1^{\circ} \pm 2,25^{\circ}$ $\left(0,5^{\circ}-12^{\circ}\right)$, meaning that average reduction of angle was $18,6^{\circ} \pm 11,4^{\circ}\left(4^{\circ}-48,5^{\circ}\right)$. Postoperative angle nearest to $0^{\circ}\left( \pm 4-5^{\circ}\right.$ or $\left.5-10 \mathrm{PD}\right)$ was present in $31(88,6 \%)$ cases and $4(11,43 \%)$ cases had postoperative angle larger than $5^{\circ}$ or $10 \mathrm{PD}$. Table 3 .

Table 3. Preoperative and postoperative angle deviation measurements \& Stereopsis

\section{Preoperative \& postoperative angle value \& Stereopsis}

\begin{tabular}{lcc} 
& Preoperative angle & Postoperative angle \\
Mean value & 20,51 & 2,13 \\
\hline Std. Error of mean value & 2,01 & 0,39 \\
\hline Std. Deviation & 11,86 & 2,25 \\
\hline Minimum & 5,00 & 0,50 \\
\hline Maximum & 50,00 & 12,00 \\
\hline Stereovision Yes & & 22 \\
\hline Stereovison No & & 10 \\
Postoperative diplopia & & 3 \\
Total & & 35
\end{tabular}

Most patients were satisified $17(48,6 \%)$ or very satisified $13(37,1 \%)$, so the total number of satisified patients was $30(85,7 \%), 3(8,6 \%)$ patients were neutral and $2(5,7 \%)$ patients were unsatisified. Table 4.

Table 4. Postoperative patients satiscfation

\begin{tabular}{ll}
$\begin{array}{l}\text { Patinets } \\
\text { satisfaction }\end{array}$ & N \\
\cline { 1 - 2 } Very satisfated & 13 \\
\hline Satisfated & 17 \\
\hline Neutral & 3 \\
\hline Unsatisfated & 2 \\
\hline Total & 35 \\
\hline
\end{tabular}

Repeated procedure was performed in $2(5,71 \%)$ cases which is also considered a good result.

\section{DISCUSSION}

In this retrospective study we observe a total of 35 patients were included, $12(34,3 \%)$ patients were male and $23(65,7 \%)$ were female, Mean age was $34,5 \pm 13$ years (20-67 years).

A relatively small number of cases is because most of adult patients find it difficult to make a decision wrther thay should have surgery or not, but the fact is that a certain number of adult strabismus were treated concervativly with ortoptics exercises or with prisms.

Exotropia $17(\mathbf{4 8 , 6 \%})$ was more frequent than esotropia 11 ( 31,4\%), and hypertropia 7 (20\%.) cases . Amblyopia was present in 16 ( 45,7\%) cases, amblyopia was also more frequent on the left eye in $\mathbf{2 1}$ cases $(60 \%)$ ). Most common indication for operative procedure was aesthetics, total of $\mathbf{2 8}$ cases $(\mathbf{8 0}, \mathbf{0} \%)$. Success rate was defined by multiple factors like orthophoria or angle near close to $o^{\circ}$, binocular vision or stereospsis and patient satisfaction. Orthophoria or postoperative angle near to $0^{\circ}\left( \pm 4-5^{\circ}\right.$ or 5 -10 prism diopters) was present in $31(\mathbf{8 8 , 6 \% )}$ cases and 4 $(\mathbf{1 1}, \mathbf{4 3} \%)$ cases had postoperative angle larger than $5^{\circ}$ or 10 prism diopters. Preoperative angle mean value was $20,5^{\circ} \pm 11,9^{\circ}\left(5^{\circ}-50^{\circ}\right)$, postoperative angle mean

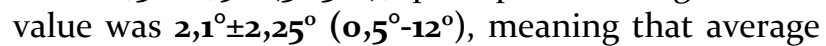
reduction of angle was $18,6^{\circ} \pm 11,4^{\circ}\left(4^{\circ}-48,5^{\circ}\right)$. Most patients were satisified $17(\mathbf{4 8 , 6 \% )}$ or very satisified 13 (37,1\%), so the total number of satisified patients was $30(85,7 \%), 3(8,6 \%)$ patients were neutral and 2 $(5,7 \%)$ patients were unsatisified.

Repeated procedure was performed in $\mathbf{2}(\mathbf{5 , 7 1} \%)$ cases which is also considered a good result.

Strabismus surgery in adults achieves satisfactory alignment with one operation in approximately $80 \%$ of patients, depending on the specific nature of the problem (1). As previously stated, approximately $20 \%$ of adults will need more than one surgical procedure to attain ocular alignment within 1o PD of straight, depending on the nature of their strabismus $(1,2)$.

Trent M.Sandercoe and all described surgical audit 
was performed on 91 adult patients, by a singular surgeon. Final measurements, on average, were taken at 16 weeks postoperatively, and patients were asked to comment subjectively on their outcome. The majority of patients were seeking surgery for combined psychosocial and cosmetic reasons (78.4\%), or combined psychosocial and functional indications (9.6\%), whereas the remainder were seeking surgery for functional indications only. $83 \%$ of patients reached the desired surgical outcome of $<10$ prism dioptres; $97.6 \%$ were satisfied or very satisfied with their surgery, with the remaining patients having a neutral standpoint, and none were dissatisfied. Finally, Adult strabismus surgery is highly successful and provides patients with a good level of satisfaction. Many patients seek surgical correction for cosmesis and psychosocial benefits (10).

Mills et al. focused on success after one surgical procedure. However, if any patient who is not satisfactorily aligned after one operation achieves successful alignment with a reoperation, it would seem axiomatic that the overall success rate of strabismus surgery would be even higher if one considered the results of a secondary surgery. In of 200 patients described 26 patients (17\%) were not satisfactorily aligned after one operation. Twenty-one of the 26 elected to have secondary surgery after which 19 (73\% of the 26) achieved a successful outcome for an overall success rate of $97 \%$ after one or two operations (11).

Scott reported a series of 892 patients he operated on and separately analyzed the 492 whose strabismus began before visual maturation, 312 whose onset was after visual maturation and 119 with complex forms of strabismus (paralytic strabismus, Brown syndrome, Duane syndrome, and dissociated vertical deviation). Scott's outcome criteria for success were different in the three groups. Patients in the frst group were primarily undergoing surgery for the purpose of improving cosmesis; for this group, he had two outcome criteria for success. One was 15 PD or less of tropia, which Scott took to represent a satisfactory cosmetic outcome, and also $8 \mathrm{PD}$ or less of tropia and $60 \%$ patints satisfaction, which would permit development of the monofixation syndrome (12).

Monte D Mills and all describe the effectiveness and safety of surgical treatment of adult patients with strabismus, and to review the reported functional benefits and complications of strabismus surgery for adults. Several large case series of adults with strabismus with successful surgical realignment rates of $68 \%$ to $85 \%$ have been reported. Functional benefits of surgical treatment are reported in many patients. These include elimination of diplopia, development of binocular fusion, expansion of binocular visual fields, and improvement of head position. Surgical complications, including new, postoperative diplopia $(1 \%-14 \%)$ or scleral perforation (o.8\%-1.8\%), occur in a minority of patients. Unplanned reoperations (subsequent strabismus procedures that were not anticipated as part of a staged treatment) were needed in up to $21 \%$ of patients in large case series of comitant strabismus, and in up to $50 \%$ of patients with thyroid ophthalmopathy. Conclusions; surgical treatment of adults with strabismus is safe and effective in improving ocular alignment. Risks include unplanned reoperation, postoperative diplopia, and scleral perforation (13).

Brianna Volk and all show psychosocial benefits of strabismus surgery as reported from postoperative patient of the 58 participating patients, $5^{1}(\mathbf{8 7 . 9 \% )}$ patients indicated positive postoperative satisfactory score; 4 (6.9\%) patients indicated negative PSS scores; and $3(5.2 \%)$ patients indicated PSS scores of zero, each of which were mentioned by at least 28 (48.3\%) patients. Preoperatively $39(67.2 \%)$ patients mentioned being self-conscious while postoperatively $3(5.1 \%)$ patients remained concerned with self-consciousness. Similarly, preoperatively $36 \quad(62.1 \%)$ patients expressed concern with their eye misalignments while postoperatively eye misalignment was a concern addressed by $3(5.1 \%)$ patients. Conclusions; Strabismus surgery benefits adult patients in many aspects including: double vision, depth perception, self-consciousness, peripheral vision, reading, head tilting, blurry vision, and eye misalignment. There is a significant psychosocial benefit in strabismus surgery (14).

Hertle described his results in 255 patients and reported successful alignment in $\mathbf{8 5} \%$ of them in series also included a heterogeneous patient mix with a large number having incomitant strabismus, vertical deviations, or multiplanar deviations (vertical and horizontal) (15).

\section{CONCLUSION}

Strabismus surgery are not exclusive for children only, they are safe and certain for adults as well, despite of age. This kind of surgeries have a very functional benefit, which includes reconstruction and restoration of motoric relations and binocularity, extension of the monocular and binocular visual field, and provide esthetic and psychological benefit. Work with adults gives a great personal satisfaction, because adult patients who get their strabismus treated were some of the happiest and most grateful patients.

\section{REFERENCES}

1. Kushner BJ. The Benefits, Risks, and Efficacy of Strabismus Surgery in Adults. Optom Vis Sci.2014 May; 91 (5): 102-9.

2. Kushner BJ, Morton GV. Postoperative binocularity in adults with longstanding strabismus. Ophthalmology 1992 Mar;99 (3):316-9.

3. Edelman PM1. Functional benefits of adult strabismus surgery. Am Orthopt J. 2010;60:43-7.

4. Noorden von GK, Campos EC. Examination 
of the PatientVIII. Binocular Vision and Ocular Motility: Theory and Management of Strabismus. St. Louis, MO: Mosby; 2002:211-45

5. Keltner JL. Strabismus surgery in adults; Functional and psychosocial implications. Arch. Ophthalmol.1994 May; 112 (5): 599-6oo.

6. Lal G, Holmes JM. Postoperative stereoacuity following realignment for chronic acquired strabismus in adults. J AAPOS 2002 Aug; 6(4): 233-237.

7. Mets MB, Beauchamp C, Haldi BA. Binocularity following surgical correction of strabismus in adults. J AAPOS 2004 Oct; 8 (5): 435-438.

8. Beauchamp GR, Black BC, Coats DK . The management of strabismus in adults - I. Clinical characteristics and treatment. J AAPOS 2003 Aug; 7(4): 233-240.

9. Ball A, Drummond GT, Pearce WG. Unexpected stereoacuity following surgical correction of long-standing horizontal strabismus. Can. J. Ophthalmol.1993 Aug; 28(5): 217-220.

10. Trent MS, Beukes S, Martin F. Adults with strabismus seek surgery for pyschosocial benefits, Taiwan Journal of Ophthalmology, 2014 March;4(1): 17-20.

11. Mills MD, Coats DK, Donahue SP, Wheeler DT. Strabismus surgery for adults: a report by the American Academy of Ophthalmology. Ophthalmology 200 Jun; 111 (6):1255-62.

12. Scott WE, Kutschke PJ, Lee WR. 2oth Annual Frank Costenbader LectureVadult strabismus. J Pediatr Ophthalmol Strabismus 1995 NovDec;32 (6):348-52.

13. Monte DM, Coats DK, Donahue SP, Wheeler DT. Strabismus surgery for adults, American Academy of Ophthalmology , 2004 June; 111(6): 1255-1262.

14. Volk B, Suh DW. Psychosocial Benefits of Strabismus Surgery in Adult Patients Investigative Ophthalmology \& Visual Science, 2014 April; Vol.55: 2601,doi: doi:https://doi.org/

15. Hertle RW. Clinical characteristics of surgically treated adult strabismus. J Pediatr Ophthalmol Strabismus 1998 May-Jun; 35 (3):138-45.

Scan this QR code with your mobile device for instant access to the current Issue of Acta Medica Saliniana

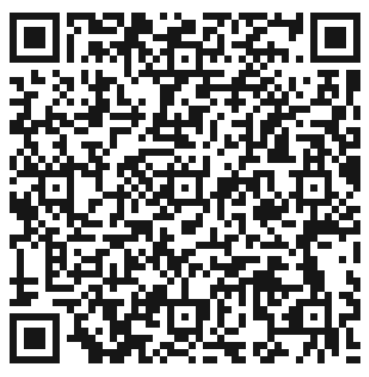

